Ausgangspunkt für den Zusammenhang zwischen Informationskompetenz und Lernen ist eine Lesart von ,in-formation', die Lernen als Bildung enthält. Informationskompetenz umfasst neben der Kenntnis effizienter Recherche- und Navigationsstrategien die Kreativität, den eigenen Informationsprozess bewusst und bedarfsgerecht zu gestalten. Der Nutzer digitaler Bibliotheken spielt als ,, information player" mit Datenbanken und Suchbegriffen, um seine Rechercheergebnisse zu verbessern. Fachspezifisch orientierte Forschungsfelder - u. a. auch die Notwendigkeit eines Blicks in die Geschichte wissenschaftlicher Information und Kommunikation dienen als Anregungen für Forschungs- und Ausbildungsthemen im Informations- und Bibliotheksbereich, deren Ziel letztendlich professionelle Informationskompetenz ist.

\title{
,In-formation' - Informationskompetenz und Lernen im Zeitalter digitaler Bibliotheken
}

\author{
Einleitung
}

„Erkannt [Gelernt] wird ... zum Ziel der In-formatio über die Welt und der Welt selber."

Ernst Bloch (1977), S.44

Die an Zahl und Komplexität zunehmenden Informationsmittel machen den Informationsdschungel immer dichter. Dies führt nicht nur in Unternehmen, sondern auch bei der wissenschaftlichen Kommunikation zu Defiziten beim Wissenstransfer. Die Industrie erwartet heute von Absolventen nicht nur fachspezifisches Wissen, sondern zusätzlich teamfähige und informationskompetente Menschen als Ergebnis der Ausbildung an den Universitäten. Die Suche nach Informationen und besonders das Finden relevanter Informationen nimmt in der modernen Informationsgesellschaft immer mehr an Bedeutung zu. Informationskompetenz ist daher die entscheidende Schlüsselqualifikation im wissenschaftlichen und beruflichen Alltag. Lebenslang Lernende müssen Informationen sicher und schnell aus dem Überangebot herausfiltern können (Fähigkeit der Bewältigung von Information: Auswahl relevanter Information, Strukturierung und Wiederauffindbarkeit). 
Collect: Sammeln benötigter Informationen und Quellen
Relate: Beziehung und Kommunikation der

$\Rightarrow$ Lernenden beim Arbeiten in gemeinschaftlichen Teams $\widehat{\imath}$

Donate: Weitergeben der Ergebnisse an Interessierte, die außerhalb der spezifischen Lernsituation stehen

\section{I}

Create: Ziel ist das Schaffen von Innovationen durch die Entwicklung anspruchsvoller Projekte

Tabelle 1: Komponenten modernen Lernens (nach Shneiderman, 2002)

Das Ergebnis zeitgemäßen Lernens ist im Optimum ein Wissensprodukt, das nicht nur für den Lernenden relevant ist (vgl. Tabelle 1). So ist das Buchprojekt, als dessen Teil dieser Beitrag publiziert wird, ein gelungenes Beispiel für solches Lernen. Das Eingangszitat von Ernst Bloch mag als Beleg dafür gelten, dass jedes Erkennen und Lernen nicht nur auf Vorhandenes gerichtet ist (,sich informierend über bereits Formiertes"), sondern auch praktische Veränderung für die Zukunft enthält, als ,,aktive In-formation eines Wirklichen selbst" (Bloch, 2000, S.238). ${ }^{1}$ Der Umgang mit Information ${ }^{2}$ umfasst also auch Elemente der Bildung und ist Teil eines jeden Lernprozesses. Lernen benötigt Informationskompetenz beim Lernenden. Neben der Kenntnis effizienter Recherche - und Navigationsstrategien umfasst Informationskompetenz vor allem die Kreativität, den eigenen Informationsprozess bewusst und bedarfs-

1 Es ist vielleicht nicht nur eine zufällige Assoziation, wenn sich Blochs grundlegende philosophische Kategorie der Möglichkeit (als ,Noch-Nicht”) in manchen theoretischen Arbeiten zum Informationsbegriff wiederfindet, so bei Hjoerland (2001), der den Begriff, ,subject” als „epistemological or informatic potential of documents” definiert, oder bei Carl Friedrich von Weizsäcker, für den Information etwas ist, was potentiell gewusst werden kann (Capurro \& Hjoerland, 2003, S.362). Dokumente als dingliche Manifestationen von Inhalten enthalten informative Möglichkeiten unabhängig davon, ob diese erkannt werden oder nicht.

2 Charakteristisch für das Konzept Information ist, dass seine Realität plausibel und nützlich in vielfältiger, oft entgegengesetzter Art und Weise - auch in Abhängigkeit von der Fachdisziplin bestimmt werden kann. Für eine Auseinandersetzung mit dem Problem ,Information' siehe Raber (2003), Klemm (2003) und Capurro \& Hjoerland (2003). Letztere weisen darauf hin, dass Konzepte wie Zeichen, Text, Dokument oder Wissen und deren Beziehung untereinander vielleicht zufriedenstellendere theoretische Bezugspunkte liefern können (S.350). Die Geschichte des Gebrauchs eines Wortes wie ,Information' liefert Erkenntnis über Zeitströmungen, den theoretischen und praktischen Hintergrund der ,Nutzer' dieses Begriffs und damit über die unterschiedliche Bedeutung, die diesem Begriff zugewiesen wird. 
gerecht zu gestalten. Letztlich führt aber nicht Information allein, sondern erfolgreiches Lernen zu Wissen, das bewusste Erkenntnis und bewusstes Verstehen (Kontext, Erfahrungen) umfasst und im Gedächtnis zugreifbar sein sollte (Marcum 2002). Noch sind Studien zum Zusammenhang zwischen Informationssuche und Lernerfolg relativ selten. ${ }^{3}$

\section{Digitale Bibliotheken und Lernen}

„Librarie-keepers ... ought to becom Agents for the advancement of universal Learning. ... his work then is to bee a Factor and Trader for helps to Learning."

John Dury (1650), S.17-18

'Digitale Bibliothek" ist ein sehr unterschiedlich verwendeter Begriff. Als Digitale Bibliothek wird heute oft eine einheitliche Präsentationsform als Portal zu digitalen Objekten, Dokumenten oder Datenquellen bezeichnet (Beispiel: Digitale Bibliothek NRW). Auch ein geordnetes und erschlossenes System digitaler Objekte trägt manchmal diese Bezeichnung. Die Digitale Bibliothek als Institution ist Synonym für die Bibliothek der Zukunft, als Gesamtheit aller Objekte/Dokumente und Dienstleistungen, die aus Sicht der heutigen Bibliothek vorstellbar sind.

Digitale Bibliotheken bestehen aus den digitalen Objekten selbst. Dazu kommen Metadaten zu digitalen und konventionellen Dokumenten sowie der Zugriff auf externe Datenressourcen (Fachdatenbanken, Suchmaschinen, Virtuelle Fachbibliotheken, usw.). Auch Personalisierungskomponenten (MyLibrary: Kundeninformation, Gespeicherte Suchen, DauerauftragsSuchen), lokale Serviceangebote (elektronische Semesterapparate, virtuelle Auskunft in Form von Online Tutorials, Chat-Service oder E-Mail-Service,...) sowie zusätzliche Community-Komponenten zur Kommunikation unter den Nutzern (z. B. im Rahmen von Computer-Supported-Cooperative-Work-Systemen) sind Teile digitaler Bibliotheken.

Bibliotheken widmen sich verstärkt nicht mehr nur der qualitativ abgesicherten, dauerhaft archivierungswürdigen Publikation, sondern öffnen sich z. B. im Rahmen der Lehre (E-Learning) auch zeitlich vergänglicheren Lernmaterialien und Produkten des Lernens an den Universitäten (Lerntexten, Projektberichten, Studien-, Abschlussarbeiten, Studierenden-Portfolios ...). Der Output des Lernens, der heutzutage in der Regel elektronisch vorliegt, führt zu einer digitalen Bibliothek von Lernobjekten. Das neue Gewicht von Lernen

3 Eine Ausnahme bildet Limberg (1999), die nachweist, dass der Inhalt die Informationssuche beeinflusst und dass die Art, wie Informationssuche vom Lernenden gesehen wird, direkt auf das Lernergebnis einwirkt. 
und Lehre in Relation zur Forschung, die Tendenz von der Wissensvermittlung zur Kompetenzvermittlung sowie die Veränderung des Lernens durch die elektronischen Möglichkeiten haben großen Einfluss auf Universitätsbibliotheken. Nach einer stark objekt- bzw. systembezogen Sicht auf digitale Bibliotheken wird immer stärker eine menschen- und kundenbezogene aktuell, die die Heterogenität und Vielfalt digitaler Bibliotheken und deren Oberflächen und damit die für den Nutzer vorhandene Problematik betont.

Die Bewältigung komplexer Systeme, wie sie z. B. auch Informationssysteme in digitalen Bibliotheken darstellen, kann nach Buckland \& Florian (1991) prinzipiell auf vier verschiedene Arten erfolgen (Tabelle 2).

\begin{tabular}{|l|l|l|}
\hline & $\begin{array}{l}\text { Verbesserung der } \\
\text { Nutzer-Kompetenz }\end{array}$ & $\begin{array}{l}\text { Reduktion der Notwen- } \\
\text { digkeit von Kompetenz } \\
\text { beim Nutzer }\end{array}$ \\
\hline $\begin{array}{l}\text { Veränderung der } \\
\text { System-Umgebung }\end{array}$ & $\begin{array}{l}\text { (1) Förderung der } \\
\text { Nutzerkompetenz }\end{array}$ & $\begin{array}{l}\text { (3) Vereinfachung der } \\
\text { Aufgabe oder Delegation } \\
\text { an einen Informations- } \\
\text { assistenten, -vermittler } \\
\text { bzw. -spezialisten }\end{array}$ \\
\hline $\begin{array}{l}\text { Veränderung } \\
\text { innerhalb des Systems }\end{array}$ & (2) Verbesserung der & $\begin{array}{l}\text { (4) Delegation der } \\
\text { Aufgabe an das System } \\
\text { oder Verbesserung des } \\
\text { Systems }\end{array}$ \\
\hline
\end{tabular}

Tabelle 2: Zur Bewältigung komplexer Systeme

Hilfe-Funktionen von Informationssystemen (2) werden viel zu wenig genutzt. Die Tendenz zur bewussten Delegation von Informationsarbeit (3) ist eher abnehmend. ${ }^{4}$ Dagegen ist für die Verbesserung von Informationssystemen (4) hinsichtlich der Oberflächen, der Nutzung von Methoden der künstlichen Intelligenz bzw. des linguistisch-maschinellen Retrievals in der Zukunft noch einiges zu erwarten. Trotzdem ist beim Nutzer der Systeme auch in Zukunft eine erhebliche eigene Kompetenz notwendig (1), z. B. hinsichtlich der Bewertung der Informationssysteme, der Rechercheergebnisse usw.

Die Bibliothek der Zukunft unterstützt nicht nur die Bereitstellung von Wissen, sondern wird selbst Teil der Forschung und des Lernens. Beratung und Medien werden bei Bedarf am ,Point of Use', im Labor oder in der Lehrveranstaltung, angeboten. ${ }^{5}$ Beim E-Learning können gerade Bibliotheken

4 Siehe jedoch Kuhlen (1999), der auch die Delegation an maschinelle „Informationsassistenten” einbezieht.

5 Ein Online-Tutorial, wie in Abschnitt 3 skizziert, kann dies unterstützen. 
wichtige Ansprechpartner z. B. für Fragen des Geistigen Eigentums oder für die Administration von Lernmanagementsystemen sein (Lynch, 2002). Typische Aufgaben von Bibliotheken im Rahmen elektronischen Lernens können die Digitalisierung von Lernmaterial z. B. im elektronischen Semesterapparat, die Beschäftigung mit Metadaten von Lernobjekten, das Angebot eines Virtual Reference Desk, die simple technische Unterstützung etwa durch die Ausleihe von Laptops oder PDAs oder auch die Anpassung von Bibliotheksservice an das Mobile Computing (PDAs, Handys, WLAN) sein.

Roes (2001) spricht sogar von einer Wiederentdeckung der Bibliothek beim Lernen und von der Notwendigkeit, dass Universitäten ihre Strategien zur Weiterentwicklung und Verbesserung des Lernens und Lehrens mit ihren Konzepten für ihre Bibliotheken, Rechen- und Medienzentren verknüpfen müssen.

\section{Schaffung eines Problembewusstseins im Bereich Informationskompetenz}

„Learning how to use databases is not only a technical but primarily a problem of social , hermeneutics' ${ }^{\text {( }}=$ the ability to ask critical questions, instead of just believing what is written or programmed or stored)."

Rafael Capurro (1990), S.132

Informationskompetenz wird von Loyd (2003) auch als Meta-Kompetenz bezeichnet, die das Erlernen neuer Fähigkeiten und neuen Wissens erst ermöglicht. Sheila Webber (2003) und Bill Johnston liefern eine ganzheitliche Definiton von Informationskompentenz als ,the adoption of appropriate information behaviour to identify, through whatever channel or medium, information well fitted to information needs, leading to wise and ethical use of information in society." Borgmann (2003, S.103ff) beschreibt modellartig drei Formen von Wissen und Fähigkeiten, die bei einem Rechercheprozess in elektronischen Datenbanken eine Rolle spielen: Neben konzeptionellem Wissen zum Prozess der Informationsgewinnung selbst, aber auch zur Informationsumgebung und zum Gebrauch von Vokabularien spielen semantisches und syntaktisches Wissen - d.h. hier Vertrautheit mit dem Leistungsvermögen der meisten Suchsysteme (logische Verknüpfungen, Anzeigemöglichkeiten usw.) und die konkreten Aktionsmöglichkeiten und Kommandos spezieller Systeme - sowie die grundlegenden technischen Fähigkeiten beim Umgang mit dem Computer eine Rolle. Dazu kommen dann noch (Fach-)Kenntnisse zum Themenbereich der Suche, ohne die weder die "richtigen" Suchbegriffe genutzt noch die Ergebnisse hinsichtlich Relevanz beurteilt werden können. 
Jeder nutzt ein Informationssystem auf seine spezielle, individuelle Art und Weise. Wichtig sind solche Fragen: Wie informiere ich mich eigentlich? Warum lohnt es sich, Zeit in das Erlernen von Recherchestrategien zu investieren? Was ist überhaupt eine Suchstrategie? Wichtiger als die Erläuterung spezifischer Systeme ist es, inhaltlich den Schwerpunkt auf Konzepte und Strategien zu legen. Notwendig sind Beratung und Hilfestellung (Consulting) zur selbstgesteuerten Optimierung der Informationskompetenz sowie die Reflexion über den eigenen Lernprozess und dessen Fortschritte. Ohne Orientierungswissen gibt es keine Wissensgesellschaft, sondern nur eine Informationsgesellschaft (Mittelstrass, 2001).

Die Notwendigkeit eines Problembewusstseins im Bereich Informationskompetenz demonstriert auch folgendes Beispiel: Eine Wissenschaftlerbefragung zum Informationsverhalten im Rahmen der Arthur D. Little-Studie (2001) ergab eine große Unsicherheit über das Ergebnis bei der Recherche nach Informationen. Hier ist deutlich $\mathrm{zu}$ machen, dass diese Unsicherheit einerseits Teil des Informationsprozesses selbst ist (Kuhlthau, 2004), andererseits Situationen der Unsicherheit in elektronischen Informationssystemen generell vorhanden sind, z. B. hinsichtlich der Qualität der Information, des Besitzes von Wissen, der Begrenzung des Zugriffs und der Delegation an Informationsassistenten (Kuhlen, 1999).

Wichtig im Sinne von Ernst Bloch und Walter Benjamin ist das ,Unscheinbare' als das ,Nicht-Scheinbare' also ,Wirkliche', sind die „Mikromomente” (Bruce, 2002) des Suchens nach Information und der Nutzung von Informationssystemen. Nicht ,der' Kontext, ,der' Nutzer oder ,das' System sind real, sondern von realer Bedeutung sind ,usings', die reale Nutzung eines spezifischen Systems durch einen spezifischen Nutzer in einem spezifischen Kontext. ${ }^{6}$

Kritische Stimmen zum „Nutzer”-Konzept (Schwarzwalder, 2000) fragen, ob interessierte ,Non-professionals' wirklich motiviert sind, ein hohes Level von Wissen über die Nutzung von Datenbanken zu erreichen. Im Zeitalter der ,Aufmerksamkeits-Ökonomie' zähle allein die Fähigkeit des InformationsBereitstellenden, seine Kunden zu erreichen, wobei Kontexte wichtiger als die

6 Dabei hat jeder Nutzer schon eine Reise bzw. seine Geschichte („Story“) hinter sich, die genau zu diesem spezifischen ,using” führt (Dervin, 1996). Dies passt zu einem Aufsatz von J. Purdue (2003), der das gängige auf Standards basierende Konzept von ,information literacy” kritisiert und Informationskompetenz wie die Forschung selbst als Teil selbst erzeugter Pfade der

Entdeckung sieht: ,Where have you come from? And where are you going?” (S.659) Eine gute Illustration dieses Ansatzes stellt das College-Buch von Watts (2003) dar, das aufgeteilt in fünf „Journeys" Studierende beim Weg durch das Studium begleitet. Ausgehend vom eigenen

Hintergrund und dessen Bedeutung für das eigene Lernen wird persönliches und wissenschaftliches Wissen verglichen und ein Verständnis von Wissenschaft als Diskurs im Laufe der Zeit sowie eine ganzheitliche Informationskompetenz dadurch erreicht, dass Studierende selbst zur Forschung beitragen, ihr Wissen mit ihrer Studiengemeinschaft teilen und über ihre Lernprozesse reflektieren. 
Inhalte seien. Zwar vereinfachen neue Oberflächen die Suche gleichzeitig führt die Vielfalt der Datenbanken zum Problem der Auswahl und Bewertung der Informationsangebote, zu dessen Bewältigung es ebenfalls der Informationskompetenz bedarf.

Auch Sheila Webber (2003) betont die Bedeutung des Kontextes bei der Informationssuche. Für sie ist Informationskompetenz weder ein Prozess noch ein Produkt, sondern ein Fachgebiet (,subject”), das sich nicht auf messbare Fähigkeiten und abfragbare Kenntnisse beschränken lasse. Es ist ein weites Spektrum an Beispielen zur Informationskompetenz-Förderung - von vollständigen Curricula bis hin zu Lernobjekten - wünschenswert, welche auf Erfahrungen des Informationsverhaltens und des Kontextes der Lernenden beruhen, Motivation bieten und pädagogisch gut strukturiert sind. Nur so lässt sich die ganze Komplexität von Informationskompetenz abbilden.

\section{Der Nutzer als ,information player' - Spielen(d) lernen}

„Clearly a major part of their [information professionals" role will be understanding, counselling, training and standing in for the i-players."

Nicholas \& Dobrowolski (2001), S.521

Angesichts ständig wechselnder Oberflächen im vielfältigen Datenbankangebot ist es für Recherchierende notwendig, Datenbanken selbstständig zu erkunden. Der Nutzer muss lernen, mit Datenbanken zu spielen, das heißt, diese im Hinblick auf ihre spezifischen Eigenschaften zu erkunden. Wie ist Boolesches Retrieval implementiert? Welche Suchfelder gibt es? Welches Wildcard-Symbol kann wie benutzt werden? Für all diese Fragen ist ein Bewusstsein zu schaffen! Erst dann kann der Nutzende in einer Datenbank quasi spielerisch herausfinden, wie die jeweilige Datenbank optimal zu nutzen ist. Wichtig ist zusätzlich der spielerische Umgang mit Suchbegriffen (Berücksichtigung von Synonymen, Quasi-Synonymen, Oberbegriffen, kontrolliertem Vokabular usw.), wenn man zur Erkundung einer Datenbank mit Quick \& Dirty-Recherchen anfängt und später sicher sein will, dass man nicht allzu viel Relevantes übersehen hat.

Albert Bilo (2003) hat zu Recht darauf hingewiesen, dass bei der Informationssuche auch assoziative, „,suboptimale” Wege zum Erfolg führen können. Die nun in die Universitäten kommende Google-Generation besitzt andere, neue Souveränitäten im Umgang mit Internet und Computer. Da soeben das Spielerische betont wurde, erschien es der Universitätsbibliothek der Technischen Universität Hamburg-Harburg (TUHH) sinnvoll, ein Tutorial zur Förderung von Informationskompetenz mit spielerischem Charakter zu entwickeln (Hapke \& Marahrens, 2004). Auch der große Zulauf zu 
Computerspielen lässt vermuten, dass ein spielerischer Ansatz hier sinnvoll sein kann. Optimal bei entsprechend vorhandenen Ressourcen wäre sicher die Entwicklung eines Strategie- oder Abenteuer-Computerspiels. ${ }^{7}$ Im Mittelpunkt moderner Nutzer-Untersuchungen im Informationsbereich steht immer mehr das Informationsverhalten in Alttag und Freizeit. Nicholas \& Dobrowolski (2001) schlagen als Ersatz für den Begriff „Nutzer” sogar den Terminus „Information Player” vor. Heutige Kunden von Informationssystemen seien interaktiv, sozial, freizeit- und wettbewerbs-betont, Eigenschaften, die auch Spieler haben. Der Unterschied zwischen Konsument und Sucher sei gering. Der Begriff helfe, den Mythos zu begraben, dass die Suche in Datenbanken eine rein wissenschaftliche Tätigkeit ist. Vorgeschlagen wird ein Verständnis von Information Professionals als Trainer und Coaches von Information Literacy.

Bisher in Deutschland vorhandene Tutorials zur Vermittlung von Informationskompetenz ${ }^{8}$ beziehen sich auf lokale Bibliothekskataloge oder enthalten kaum aufgabenorientierte, interaktive Elemente. Gabi Reinmann-Rothmeier (2003) hebt als wichtige Komponenten des elektronischen Lernens Emotionen wie z. B. Neugier (Interesse, Spiel-Konzept) hervor. Selten wird z. B. die sinnliche Wahrnehmung beim Recherchieren, das konkrete Umgehen mit Unwägbarkeiten und deren Voraussehen so klar beschrieben wie von Sabine Pfeiffer (1999, S.96-108). Erfahrung, Wissen, Denken und Gefühl spielen alle beim Recherchieren eine große Rolle. Die kreative Nutzung von Werkzeugen im Rahmen gestaltender Aufgaben - nämlich dem Create und Donate aus Tabelle 1 - ist wichtiger als ein strukturiertes Edutainment-Angebot.

\section{Was wird im Boole'schen Restaurant serviert?}

Suchen Sie im Katalog der TUHH-Bibliothek einmal nach der Zeitschrift „Science“ oder nach Literatur, die irgendwie mit der Insel ,Java“ zu tun hat! Haben Sie schon einmal bei der Recherche in einer Datenbank das Gefühl gehabt, die Möglichkeiten des Systems nicht richtig ausnutzen zu können? Kennen Sie die wichtigsten Datenbanken Ihres Fachgebiets?

Sind Sie neugierig geworden?

Schauen Sie doch mal auf unseren Prototyp unter discus.tu-harburg.de!

Mit obigem Kasten wirbt die Universitätsbibliothek der TUHH für ihr Projekt Developing Information Skills \& Competence for University Students (DISCUS). ${ }^{9}$ Dieses deutsch- und englischsprachige Online-Tutorial fördert die

7 Vergleiche etwa das Produkt Informaticus, Stuttgart, 2003, siehe auch Meier \& Seufert (2003)

8 Rauchmann (2003) gibt einen umfassenden Überblick zur Gesamt-Entwicklung im Bereich

Informationskompetenz und Bibliotheken in Deutschland.

${ }^{9}$ Weitere Information über das Projekt auf der Website der Universitätsbibliothek der TUHH, 
Schaffung eines Problembewusstseins im Bereich Informationskompetenz bei Studierenden, Lehrenden und Forschenden. DISCUS soll die Nutzung elektronischer Fachinformation an Hamburger Hochschulen optimieren und dadurch erfolgreichere Forschungsergebnisse und Studienabschlüsse ermöglichen. Das unter Einsatz von Mitteln des Senates der Freien Hansestadt Hamburg $\left(\mathrm{ELCH}^{10}\right)$ entstehende E-Learning-Tutorial bildet ein Beispiel für Aktivitäten, als Bibliothek im Ökosystem des Lernens an einer Universität auch in elektronischer Umwelt wahrgenommen zu werden. Neben dem als Prototyp realisierten Modul zu Navigations- und Recherchestrategien im Rahmen systematischer Informationssuche ist zukünftig auch an Module zur Bewertung („Critical Skills“), zur Organisation (Dokumentenmanagement) und Präsentation von Information, zum elektronischen Publizieren (AutorenBeratung: Schreiben, Zitieren, Formatieren) sowie zu sozialen Aspekten von Informationssystemen (Geistiges Eigentum, Urheberrecht, Informationspolitik, kulturelle Überlieferung) gedacht, um letztendlich alle Dimensionen der Informationskompetenz zu berücksichtigen. Fachspezifische Module zur Verfahrenstechnik und Biotechnologie vermitteln über praxisorientierte Aufgaben Erfahrungen in der Nutzung einschlägiger Informationsquellen (Aufsatz-, Stoff-, Patent-Datenbanken).

Die unterschiedliche Verwendung der Boole'schen Operatoren im Alltagsgebrauch und bei der Recherche in einer Datenbank demonstriert im Tutorial das Boole'sche Restaurant, in dem die Gerichte als Datensätze in einer „Datenbank“ fungieren, während die Recherche-Oberfläche durch die Speisekarte dargestellt wird. Nach Auswahl

\begin{tabular}{|c|c|c|}
\hline \multicolumn{3}{|c|}{ In Boole's Restaurant } \\
\hline \multicolumn{2}{|c|}{ Klicken Sie bitte auf Ihre Bestellung! } & Was bringt der Wirt? \\
\hline \multicolumn{2}{|c|}{ Gasthaus bei $\mathrm{Bo} / \mathrm{e}$} & $\begin{array}{l}\square \text { Gemüse-Suppe } \\
\square \text { Kartoffel-Suppe } \\
\square \text { Kartoffel-Salat }\end{array}$ \\
\hline Gemüse UND Suppe & 5,50 & $\square$ Grüner Salat \\
\hline Suppe ODER Salat & $3,--$ & $\begin{array}{l}\text { Tomaten-Salat } \\
\text { Kartoffel }\end{array}$ \\
\hline Suppe NICHT Gemase & 3,-- & $\square$ Nudel \\
\hline $\begin{array}{l}\text { Suppe UND Salat UND } \\
\text { Kartoffel }\end{array}$ & $10,--$ & $\begin{array}{l}\square \text { Nudel-Salat } \\
\square \text { Gemüse }\end{array}$ \\
\hline $\begin{array}{l}\text { Salat UND (Kartoffel ODER } \\
\text { Nudel) }\end{array}$ & $10,--$ & $\begin{array}{l}\square \text { Suppe } \\
\square \text { salat }\end{array}$ \\
\hline $\begin{array}{l}\text { Salat ODER Suppe UND } \\
\text { (Gemüse ODER Kartoffel) }\end{array}$ & $15,-$ & \\
\hline $\begin{array}{l}\text { (Salat ODER Suppe) UND } \\
\text { (Gemüse ODER Kartoffel) }\end{array}$ & $15,-$ & \\
\hline Überprüfen & & \\
\hline
\end{tabular}
eines Gerichtes auf der Speisekarte muss man entscheiden, welche Gerichte

www.tub.tu-harburg.de/index.php?id=418 . Ein Prototyp ist unter discus.tu-harburg.de öffentlich verfügbar.

${ }^{10}$ E-Learning-Consortium Hamburg, siehe www.e-learning-hamburg.de und Multimedia Kontor Hamburg, www.mmkh.de 
der Kellner bringen wird. Interaktiv, selbsttätig und spielerisch kann so Kompetenz vermittelt und die Boole'sche Logik bewusst gemacht werden.

\section{Fachspezifische Forschungsfelder im Informations- und Bibliotheksbereich und der Blick auf die Geschichte}

Ein wichtiger Zweck von Geschichtsschreibung ist „Entfamiliarisierung ..., eine Form der Entfremdung, die das ehemals Vertraute fremd macht und das ehemals Natürliche willkürlich".

Peter Burke (2001), S.10

Lernen und Forschen werden in der Zukunft kaum zu trennen sein. Anregungen für wichtige Lern- und Forschungsthemen im Informations- und Bibliotheksbereich, deren eigentliches Ziel letztendlich professionelle Informationskompetenz ist, bieten fachspezifisch orientierte Forschungsfelder nach Birger Hjoerland (2002) - u. a. auch die Notwendigkeit eines Blicks in die Geschichte wissenschaftlicher Information und Kommunikation (,Zukunft braucht Herkunft").

Hjoerland sieht Nutzer als Teil einer fachlichen Diskussions- und DiskursGemeinschaft mit eigenen kulturellen und sozialen Strukturen, die ein gemeinsames Vokabular und eine typische Informationspraxis teilt. Diese „sozio-kognitive” Sicht (Hjoerland, 2004) betont die Bedeutung der historischen Entwicklung dieser Gemeinschaften und ihrer Kommunikationsprozesse, verbunden mit einer Untersuchung der Strukturen, Dokumenttypen und Institutionen wissenschaftlicher Kommunikation und Information. Weitere Elemente dieses „domain”-analytischen Ansatzes sind: die Erstellung von Literaturführern und „subject gateways”, von speziellen Klassifikationen, Thesauri sowie Indexierungs- und Rechercheinstrumenten, die Durchführung empirischer Nutzerstudien sowie bibliometrischer, kritisch-erkenntnistheoretischer und terminologischer Studien.

Die Geschichte wissenschaftlicher Information und Kommunikation als eigenständiges Forschungsgebiet umfasst die Geschichte wissenschaftlicher Publikations- und Kommunikationsmedien sowie die Entwicklung ihrer Erschließung in ,Memory'-Institutionen (Archive, Bibliotheken, Museen) und Informationssystemen (Hapke, 2001). Sie ist auf enge Zusammenarbeit mit der Wissenschafts- und Technikgeschichte - heute besonders der ComputerGeschichte - , sowie mit der Bibliotheks-, Buch- und Mediengeschichte angewiesen. Gerade das Wachstum des Internets stellt althergebrachte Vorstellungen von Begriffen, wie z. B. Dokument, Format, Publikation, 
Zeitschrift, Sammlung oder Bibliothek, in Frage und verbindet damit die zukünftige Entwicklung mit der Überlieferung.

Auch in der Wissenschafts- und Technikgeschichte selbst sind als Folge einer ,kulturellen Wende' Forschungsgegenstände wie Kommunikationsprozesse und Werkzeuge bzw. Instrumente wissenschaftlicher Forschung immer wichtiger geworden (Hard, 2003). Disziplinen werden als soziale und intellektuelle Infrastruktur der Wissenschaft gesehen, wo Aushandlungsprozesse und Macht und damit Kommunikationsprozesse entscheidend sind (Schirrmacher, 2003). Untersucht werden in einer „ökologischen” Betrachtungsweise die Wechselwirkung und Lebensbedingungen der unterschiedlichen Spezies von Wissenschaftlern in ihren Ökosystemen („Milieus”) bzw. sozialen disziplinären Gemeinschaften. Wissenschaft als kulturelles Phänomen wahrzunehmen bedeutet, „die historische Dimension des Wissens und seiner Repräsentationsformen, seiner grundlegenden Kategorien und Medien, seiner Praktiken und kulturellen, sozialen und ökonomischen Verwebungen ernst zu nehmen" (Hagner, 2001, S.30). Hier wird also die Bedeutung einer Geschichte wissenschaftlicher Information sichtbar. Dieser Aufwertung der materiellen Repräsentationsformen der wissenschaftlichen Gegenstände und Phänomene folgt eine Aufwertung der Bedeutung der Geschichte der Institutionen, die diese überliefern (Archive, Bibliotheken, Museen). Daher hat die jüngste Vergangenheit auch wichtige Beiträge aus kultur- und medienhistorischer Sicht auf diese Memory-Institutionen hervorgebracht (Krajewski, 2002; Ernst, 2003).

Ein historischer Zugang zu Informationssystemen ist auch noch für einen anderen Zweck wichtig: Die Behandlung der sozialen Auswirkungen des Informationszeitalters und damit auch historische Betrachtungen können ein wichtiger Teil in Lehrveranstaltungen zur Vermittlung von Informationskompetenz sein. In diesem Sinne erwähnt Breivik (1998, S.40) Michael Buckland, einen der Initiatoren der ersten Conference on the History and Heritage of Science Information Systems in Pittsburgh, Pennsylvania im Jahre 1998. ${ }^{11}$ Dieser unterscheidet zwei Traditionen innerhalb der „Landschaft“ des heutigen Informationswesens, eine basierend auf dem Dokument, die andere auf der Technik (heute dem Computer). Nur die Beachtung beider Traditionslinien ermöglichen ein vollständiges, adäquates und umfassendes Verständnis heutigen Informationsmanagements.

\footnotetext{
${ }^{11}$ Siehe Bowden [u. a.] (1999). Ende 2004 soll der Proceedings-Band der Second Conference on the History and Heritage of Scientific and Technical Information Systems 2002 in Philadelphia publiziert werden (siehe http://www.chemheritage.org/events/event-asist2002.html).
} 


\section{Fazit}

Der letzte Abschnitt schlug einen Bogen von der allgemeinen zur professionellen Informationskompetenz und damit zur Ausbildung der zukünftig im Bibliotheks- und Informationsbereich Tätigen. Da die Qualität der Information auch von den Bedürfnissen und Anforderungen der Nutzer und Wissenschaftler abhängt, ist es wichtig, die soziale Struktur und Dynamik der Wissenschaften zu verstehen, gerade um Informations-Dienstleistungen für Forschung und Lehre anbieten zu können. Folgerichtig fragt Van House (2004) nach dem Zusammenhang zwischen „Information (and Communication) Studies” und „Science and Technology Studies”.

Auch bei der Betrachtung digitaler Bibliotheken ist eine „ökologische" Sichtweise zu finden, die im letzten Abschnitt aus die Wissenschaftsgeschichte beschrieben wurde. Für O'Day und Hardi (2003) ist gerade die ÖkologieMetapher sinnvoll, um über Technik zu reflektieren. Für sie reichen die Metaphern Werkzeug, Text, ,assistant” und System nicht aus, die komplexen Abhängigkeiten zwischen Werkzeugen und Verfahren in digitalen Bibliotheken herauszuarbeiten und die Möglichkeiten der diversen lokalen Variationen $\mathrm{zu}$ betonen. Sie beschreiben die Bibliothek als InformationsÖkosystem und fragen nach Vielfalt, Lokalität, „Coevolution” von digitaler mit konventioneller Bibliothek und nach „keystone species” (Welche Rolle spielen Bibliothekare in digitalen Bibliotheken? Welche Bedürfnisse existieren für Vermittlung und Vermittler?).

Der Philosoph Gernot Böhme (1999) hat eine ,antizyklische Bildungspolitik" gefordert, die gerade das fördert, ,was nicht im manifesten Trend der Entwicklung liegt”. Dieser Beitrag versucht eine Art dialektischen Spagat zwischen Trend und Anti-Trend, zwischen Informationskompetenz und Informationsgeschichte. (Aus-)Bildung ist nicht als Vorbereitung auf das Leben, sondern selbst als Leben zu erfahren. Ein wirkliches Verständnis von Dienstleistung als ,Information Professional' für die Forschung kann nur erreicht werden, wenn Forschung als eigene Tätigkeit im Rahmen der Ausbildung erfahren wird. Die Grundfrage, was unterscheidet einen professionellen Informationsspezialisten von einem, normalen' Nutzer, muss immer wieder neu ver-antwort-et werden. Informationsver-,mitt'-lung muss sich dem sich ändernden Lern- und Kompetenzbegriff anpassen. Für das Lernen in Hochschulen und Bibliotheken wird so etwas wie eine „Ermöglichungsdidaktik” (nach Rolf Arnold in Fürnhammer, 2003) und „User (learner) empowerment” benötigt, zur Entwicklung der Potentiale der Kunden (Lernenden), zur ,information" der Nutzer. 


\section{Literatur und Internetquellen}

Arthur D. Little: Zukunft der wissenschaftlichen und technischen Information in Deutschland : Ergebnisse der empirischen Untersuchungen über das Informationsverhalten von Wissenschaftlern und Unternehmen. Zwischenbericht an das Bundesministerium für Bildung und Forschung. Oktober 2001. http://www.dl-forum.de /Initiativen/Strategiekonzept/ErsteErgebnisse1/ErsteErgebnisse2/index.html

Bilo, Albert: Elektronische Informationen in der Hochschulausbildung [Rezension], in: Bibliothek 27 (2003), S. 238-240

Bloch Ernst: Logos der Materie : eine Logik im Werden : aus dem Nachlass 1923-1949 / hrsg. von Gerardo Cunico. Frankfurt a.M., 2000

Bloch, Ernst: Tübinger Einleitung in die Philosophie. Frankfurt a.M., 1977

Böhme, Gernot: Bildung als Widerstand : was sollen die Schulen und Hochschulen lehren? Ein Versuch über die Zukunft des Wissens, in: Die Zeit, 1999, Nr. 38

Borgman, Christine L.: Designing digital libraries for usability, in: Digital library use : social practice in design and evaluation / ed. by Ann Peterson Bishop, ... Cambridge, Mass., 2003, S. 85-119

Bowden, Mary Ellen [u. a.] (Eds.): Proceedings of the 1998 Conference on the History and Heritage of Science Information Systems. Medford, N.J., 1999

Breivik, Patricia S.: Student learning in the information age. Phoenix, Ar.: 1998

Bruce, Harry: The user's view of the Internet. Lanham, Md., 2002

Buckland, Michael: The landscape of information science: the American Society for Information Science at 62, in: Journal of the American Society of Information Science 50 (1999), S. 970-974

Buckland, Michael; Florian, Doris: Expertise, task complexity and artificial intelligence, in: Journal of the American Society for Information Science 42 (1991), S. 635-643

Burke, Peter: Papier und Marktgeschrei : die Geburt der Wissensgesellschaft. Berlin, 2001

Capurro, Rafael; Hjoerland, Birger: The concept of information, in: Annual Review in Information Science and Technology 37 (2003), S. 343-411

Capurro, Rafael: Towards an information ecology, in: Information quality : definitions and dimensions / ed. by Irene Wormell. London, 1990, S. 122-139

Dervin, Brenda: Information Needs and Information Seeking : The "Search For Questions Behind the Research Agenda ; Beitrag auf dem Workshop 
„Social Aspects of Digital Libraries” an der University of California, Los Angeles, February 16-17, 1996, http://is.gseis.ucla.edu/research/dl/dervin.html Dury, John: The reformed Librarie-Keeper (1650) / Introd. by Richard H. Popkin and Thomas F. Wright. Los Angeles, 1983

Ernst, Wolfgang: Im Namen von Geschichte : Sammeln - Speichern Er/Zählen ; infrastrukturelle Konfigurationen des deutschen Gedächtnisses. München, 2003

Fürnhammer, Hans: Bibliothek - Informationskompetenz - Informationelle Autonomie : Aspekte der Positionierung einer wissenschaftlichen Fachbibliothek, in: Mitteilungen der Vereinigung Österreichischer Bibliothekarinnen und Bibliothekare 56 (2003) 2, S. 19-26

Hagner, Michael: Ansichten der Wissenschaftsgeschichte, in: Ansichten der Wissenschaftsgeschichte / hrsg. von Michael Hagner. Frankfurt am Main, 2001, S. 7-39

Hapke, Thomas; Marahrens, Oliver: Spielen(d) lernen mit DISCUS : Förderung von Informationskompetenz mit einem E-Learning-Projekt der Universitätsbibliothek der TU Hamburg-Harburg, in: Information Professional 2011 : Strategien - Allianzen - Netzwerke. 26. Online-Tagung der DGI. Frankfurt am Main vom 15. bis 17. Juni 2004. Proceedings / hrsg. von Marlies Ockenfeld. Frankfurt am Main, 2004, hier S. 203-217

Hapke, Thomas: Bausteine zur Geschichte des deutschen Informationswesens im 20. Jahrhundert - eine Vorbereitung auf die 2. Conference on the History and Heritage of Scientific and Technical Information Systems, in: Information research \& content management : Orientierung, Ordnung und Organsiation im Wissensmarkt / hrsg. von Ralph Schmidt. Frankfurt a.M., 2001, S. 498-506

Hard, Mikael: Zur Kulturgeschichte der Naturwissenschaften, Technik und Medizin, in: Technikgeschichte 70 (2003), S. 23-45

Hjoerland, Birger: Domain analysis : a socio-cognitive orientation for information science research, in: Bulletin of the American Society for Information Science and Technology 30 (2004) 3, S. 17-21

Hjoerland, Birger: Domain analysis in information science, in: Journal of documentation 58 (2002), S. $422-464$

Hjoerland, Birger: Towards a theory of aboutness, in: Journal of the American Society for Information Science and Technology 52 (2001), S. 774-778

Klemm, Helmut: Ein großes Elend : das Informationszeitalter kann sich nicht einigen über den Begriff „Information”, in: Informatik-Spektrum 24 (2003), S. $267-273$ 
Krajewski, Markus: Zettelwirtschaft : die Geburt der Kartei aus dem Geiste der Bibliothek. Berlin, 2002

Kuhlen, Rainer: Die Konsequenzen von Informationsassistenten : was bedeutet informationelle Autonomie oder wie kann Vertrauen in elektronische Dienste in offenen Informationsmärkten gesichert werden? Frankfurt a.M., 1999

Kuhlthau, Carol Collier: Seeking meaning : a process approach to library and information services. 2nd ed. Westport, Conn., 2004

Limberg, Louise: Experiencing information seeking and learning: a study of the interaction between two phenomena, in: Information Research 5 (1999) 1, http://informationr.net/ir/5-1/paper68.html

Loyd, Annemaree: Information literacy : the meta-competency of the knowledge economy? An exploratory paper, in: Journal of Librarianship and Information Science 35 (2003) 2, S. 87-92

Lynch, Clifford: The afterlives of courses on the network : information management issues for learning management systems, in: EDUCAUSE Research Bulletin, Vol. 2002, Issue 23,

http://www.cni.org/staff/cliffpubs/ECARpaper2002.pdf

Marcum, James W.: Rethinking information literacy, in: Library Quaterly 72 (2002), S. 1-26

Meier, Christoph; Seufert, Sabine: Lebenslanges (E-)Learning: Lust oder Frust? Zum Potential digitaler Lernspiele für die betriebliche Bildung, in: Weiterlernen neu gedacht. QUEM-Report H. 78 (2003), S. 179-192, http://www.abwf.de/content/main/publik/report/2003/Report-78.pdf

Mittelstrass, Jürgen: Wissen und Grenzen : philosophische Studien. Frankfurt a.M., 2001

Nicholas, David; Tom Dobrowolski: The ,information player' : a new and timely term for the digital information user, in: Handbook of information management / ed. by Alison Scammell, Association for Information Management. 8. ed. London, 2001, S. 513-522

O’Day, Vicki L.; Hardi, Bonnie A.: An ecological perspective on digital libraries, in: Digital library use : social practice in design and evaluation / ed. by Ann Peterson Bishop ... Cambridge, Mass., 2003, S. 65-82

Pfeiffer, Sabine: Dem Spürsinn auf der Spur : subjektivierendes Arbeitshandeln an Internet-Arbeitsplätzen am Beispiel Information-Broking. München, 1999

Purdue, J.: Stories, Not Information: Transforming Information Literacy, in: Portal: Libraries and the Academy 3 (2003) 4, S. 653-662 
Raber, Douglas: The problem of information : an introduction in information science. Lanham, Maryland, 2003

Rauchmann, Sabine: Die Vermittlung von Informationskompetenz in OnlineTutorials : eine vergleichende Bewertung der US-amerikanischen und deutschen Konzepte, in: Innovationsforum 2003 / hrsg. von Rolf Fuhlrott ... Wiesbaden, 2003, S.189-285

Reinmann-Rothmeier, Gabi: Die vergessenen Weggefährten des Lernens : Herleitung eines Forschungsprogramms zu Emotionen beim E-learning. Arbeitsbericht 1. Augsburg: Universität Augsburg, Medienpädagogik, 2003, http://static.cognitivearchitects.com/gems/medienpaedagogik/AB1.pdf

Roes, Hans: Digital libraries and education : trends and opportunities, in: DLib Magazine 7 (2001) 7/8, http://www.dlib.org/dlib/july01/roes/07roes.html

Schirrmacher, Arne: Die Entwicklung der Sozialgeschichte der modernen Mathematik und Naturwissenschaften und die Frage nach dem sozialen Raum zwischen Disziplin und Wissenschaftler, in: Berichte zur Wissenschaftsgeschichte 26 (2003), S.17-34

Schwarzwalder, Robert: The death of the end user, in: EContent 23 (2000) August/September, S.73-75

Shneiderman, Ben: Leonardo's laptop : human needs and the new computing technologies . Cambridge, Mass., 2002

Van House, Nancy A.: Science and technology studies and information studies, in: Annual Review of Information Science and Technology 38 (2004), S.3-86

Watts, Margit Misangyi: College : we make the road by walking. Upper Saddle River, N.J., 2003

Webber, Sheila: An International Information Literacy Certificate: opportunity or dead-end? World Library and Information Congress: 69th IFLA General Conference and Council, 1-9 August 2003, Berlin, http://www.ifla.org/IV/ifla69/papers/199e-Webber.pdf

Die angegebenen Internetadressen wurden zuletzt am 28.06.2004 aufgerufen. 